\title{
Estudios sobre alimentación y nutrición en México: una mirada a través del género
}

\author{
Sara Elena Pérez Gil-Romo, Lic en Nutr, Lic en Soc, D en Antr, ${ }^{(1)}$ \\ Silvia Díez-Urdanivia Coria, Lic en Nutr, M en Des Rural(2)
}

\author{
Pérez Gil-Romo SE, Díez-Urdanivia S. \\ Estudios sobre alimentación y nutrición en México: \\ una mirada a través del género. \\ Salud Publica Mex 2007;49:445-453.
}

\section{Resumen}

En este trabajo se reflexiona acerca de la utilización de la categoría de género en los estudios de alimentación y nutrición en México y se destaca la importancia de las mujeres como responsables de la salud y la nutrición familiar. Además, se hace una breve revisión acerca de la manera en que las mujeres han sido analizadas en las diferentes encuestas de nutrición y alimentación y se concluye que, o bien son "invisibilizadas", al igual que los hombres, como parte de los promedios per cápita, o únicamente se les considera como parte de su rol reproductor. Se destaca la importancia de incluir el género y la perspectiva de género en los estudios alimentarios, con el objeto de profundizar en el conocimiento sobre las desigualdades en alimentación entre hombres y mujeres en las diferentes etapas del proceso alimentario: decisión, provisión, preparación, distribución y consumo de alimentos, así como de las prácticas y significados que tienen los alimentos para las mujeres. Se concluye haciendo énfasis en la importancia de introducir el tema de género en los currículos académicos de las diferentes escuelas de nutrición, enfermería y medicina.

Palabras clave: género; sexo; alimentación; nutrición; México
Pérez Gil-Romo SE, Díez-Urdanivia S.

Food and nutrition studies in Mexico:

A gender perspective.

Salud Publica Mex 2007;49:445-453.

\section{Abstract}

The present paper reflects on utilization of the gender category in food and nutrition studies in Mexico. It highlights women's important role as caretakers of family health and nutrition. Briefly the authors review how women have been analysed in the different food an nutrition surveys; how gender has been effaced in the "mean per capita", and how women have been taken into account only as far as their reproductive role.This paper also outlines the importance of including the gender category and gender approach in food and nutrition studies; in order to clearly visualize the feeding inequities among men and women throughout the stages of the feeding process, i.e. decision, provision, preparation, distribution and intake. Moreover, improved understanding of women's nutrition practices and meanings that foods convey to women is advocated. In the conclusion the absolute need to articulate the gender issue in nutrition, nursing and medical academic curricula is stated.

Key words: gender; sex; feeding; nutrition; Mexico

(I) Instituto Nacional de Ciencias Médicas y Nutrición Salvador Zubirán (INNSZ). Universidad Autónoma Metropolitana Xochimilco. México, D.F.

(2) INNSZ. México, D.F.

Fecha de recibido: I3 de febrero de 2007 • Fecha de aceptado: I 3 de agosto de 2007 Solicitud de sobretiros: Sara Elena Pérez Gil Romo. Instituto Nacional de Ciencias Médicas y Nutrición Salvador Zubirán. Vasco de Quiroga 15, col. Sección XVI, I4000 Tlalpan. México, D.F.

Correo electrónico: seperezgil@laneta.apc.org 
$E^{n}$ México, al hacer una revisión sobre los diversos estudios que se han realizado en el área de la salud, diversas investigadoras e investigadores observaron que "éstos han puesto en evidencia la exclusión de las mujeres en la investigación y la falta de puntualización de las desigualdades en la atención médica que afectan primordialmente a las mujeres" ${ }^{1}$ Si bien es cierto que desde hace algunos años se pueden encontrar trabajos que analizan la mortalidad, la morbilidad, la salud sexual y reproductiva y la salud mental bajo una perspectiva de género, las reflexiones sobre lo que implica este concepto están casi siempre ausentes en la mortalidad y la morbilidad y, en general, el término de género se llega a utilizar como sinónimo de sexo, de mujeres, o de diferencia sexual. El género ha sido escasamente incorporado como una categoría de análisis en las investigaciones de nutrición y alimentación y casi siempre se aplica sólo para hacer referencia al sexo femenino.

A partir de lo anterior, en este ensayo se busca compartir algunas reflexiones acerca de la categoría de género y su aplicación en las investigaciones de alimentación y nutrición. Para cumplir con ese objetivo, se dividió el trabajo en varios apartados: en el primero se discute la importancia de las mujeres como responsables de la salud y la alimentación de toda la familia. En el segundo se hacen reflexiones sobre la perspectiva de género y su relevancia en los estudios de salud. Posteriormente, se hace una breve revisión acerca de la forma en que las mujeres han sido analizadas en los principales estudios de alimentación y de nutrición en el país y, finalmente, se discute la aportación de utilizar la categoría y la perspectiva de género en las investigaciones sobre esta temática, proporcionando algunos ejemplos de sus aportaciones con un estudio sobre lactancia materna realizado en comunidades del estado de Oaxaca.

\section{Las mujeres y su responsabilidad frente a los otros y otras: la salud y la alimentación}

Las mujeres son el centro de atención de gran parte de los estudios y programas aplicados de nutrición y alimentación en México, en tanto que se consideran uno de los componentes fundamentales de la salud infantil. Son el foco de interés en términos de su reproducción en el embarazo y la lactancia, momentos en que aumentan sus requerimientos de energía y de ciertos nutrimentos, lo cual, aunado a los cortos intervalos intergenésicos, al intensivo cuidado de los hijos (reproducción social) y a una mala alimentación, hace de la población femenina uno de los grupos con mayor riesgo. En años recientes, las mujeres que pasan por el climaterio y la menopausia también se han convertido en las unidades de análisis de varias investigaciones, debido a sus cambios en los requerimientos de varios nutrimentos y a la relación entre algunas patologías y la ingesta de ciertos alimentos.

No obstante, algunas investigadoras alertan que caracterizar a la mujer esencialmente por sus funciones reproductivas, esto es, sólo "en tanto madre", es lo que ha llevado a las/los profesionales de la salud y la nutrición a estudiar casi en forma exclusiva el estado nutricio de las mujeres-madres durante ciertos momentos del proceso reproductivo, sin percatarse de que esta visión puede distraer la atención de otros elementos de discriminación y desventaja. ${ }^{2,3}$ La construcción de la identidad femenina centrada en la maternidad se define en lo femenino como un "ser para otros", ${ }^{4}$ lo cual llega a tener implicaciones en la autoestima, la valoración social y la capacidad para tomar decisiones y actuar en su propio cuerpo, su vida y su salud, incluida su alimentación. La mujer es, sobre todo, quien nutre y alimenta a los demás y esa parte de la construcción de la identidad cuestiona la posibilidad de cuidar su propio bienestar. De lo anterior se desprende la necesidad de contrarrestar el sesgo generalizado de restringir la salud de la mujer al ámbito reproductivo-materno.

Los acercamientos a la salud de la mujer que desborden la salud reproductiva como único foco de análisis han sido propiciados, de acuerdo con Ravelo, ${ }^{2}$ por la incorporación de planteamientos originados en las ciencias sociales. Lo anterior contrasta con la perspectiva biomédica sobre la denominada salud materno-infantil y con las políticas públicas que consideran principalmente a las mujeres como categorías poblacionales. El estudio de la salud de las mujeres, que se había concentrado sobre todo en el campo de las ciencias biológicas, pasó a formar parte del conocimiento de las ciencias sociales en particular de la sociología, de la antropología médica y de las ciencias denominadas sociomédicas, como la salud pública, la salud comunitaria y la medicina social. Entre los principales logros, producto de estos nuevos abordajes, está el interés por la relación salud y género, o salud de las mujeres, que rebasó el marco de la reproducción biológica al incorporar aspectos socioculturales, económicos y políticos. En este sentido, el campo de la salud femenina se ha ampliado, diversificándose las temáticas y los acercamientos, entre ellos el de la nutrición y alimentación, tema central de este trabajo. A continuación se recapitulará en lo que es el género y la perspectiva de género.

Como ya se ha dicho, el concepto de género se utiliza a menudo como sinónimo de sexo, de mujeres, o de diferencia sexual, y la perspectiva de género sólo para hacer referencia al sexo femenino. Lamas señala ${ }^{5}$ "que aunque usar género o perspectiva de género como mujeres o perspectiva que toma en cuenta la existencia 
de las mujeres es cuestionable, desde un punto de vista conceptual y político es útil, pues conduce al rechazo de términos neutros como paciente, derechohabiente y ciudadano". Al ampliar lo anterior, esta autora comenta que la sustitución de mujeres por género se da en todas partes, y que entre los hispanoparlantes tiene una justificación de peso, ya que en castellano se habla de las mujeres como el género femenino, por lo que es fácil deducir que hablar de género o de perspectiva de género es referirse a las mujeres o a la perspectiva del sexo femenino.

Scott ${ }^{6}$ explica cómo, en los ochenta, "la búsqueda de legitimidad académica" llevó a las estudiosas feministas a sustituir mujeres por género. Resulta interesante lo señalado por esta investigadora cuando dice que "el empleo de género trata de subrayar la seriedad académica de una obra, porque género suena más neutral y objetivo que mujeres [...] género no comporta una declaración necesaria de desigualdad o de poder, ni nombra al bando contrario (hasta entonces invisible) oprimido ... género incluye a las mujeres sin nombrarlas y así parece no plantear amenazas críticas". Para Scott el género designa también las relaciones sociales entre los sexos y la forma de situarse en el debate teórico. En otros términos, la categoría de género tiene dos dimensiones que son de utilidad, según el ámbito desde donde se vea: por un lado se encuentra la dimensión teórica que permite conceptualizar la relación social a partir de su ubicación en una estructura sexual históricamente determinada, esto es, una estructura de relaciones y significaciones donde lo femenino y lo masculino han tenido algunos cambios a lo largo de la historia; $y$, por el otro, existe una dimensión que se ubica en el terreno de la praxis, como eje rector de la relación, debido a que, en términos reales, constituye una relación social con implicaciones diferentes para cada uno de los géneros, lo que ha generado situaciones de desigualdad social.

El uso de la categoría de género implica que, dependiendo de la disciplina de que se trate, se formulará la interrogante sobre ciertos aspectos de las relaciones entre los sexos o de la simbolización cultural de la diferencia sexual. ${ }^{5,7}$ Desde la antropología, la definición de género o de perspectiva de género alude al orden simbólico con que una cultura dada elabora la diferencia sexual. Goldsmith, ${ }^{7}$ al discutir sobre los debates antropológicos relacionados con los estudios de la mujer, argumenta que los llamados estudios sobre las mujeres desencadenaron un profundo cuestionamiento dentro de la disciplina de la antropología. Al poner en duda la validez científica de investigaciones que se habían llevado a cabo con grandes premisas y/o sesgos etnocéntricos, androcéntricos o clasistas, se hizo necesaria, según esta autora, la reconsideración de algunos de los postulados básicos de esta disciplina. Así pues, en un intento por abordar la relevancia de este concepto dentro de los estudios de alimentación y nutrición, a continuación se rescatarán algunos aspectos sobre el género que son importantes en esta aproximación y que, por otro lado, desde nuestra perspectiva, resultan novedosos en las investigaciones sobre dichos temas.

Lamas $^{5}$ señala que si tuviera que elegir un concepto que distinguiera a la antropología de las demás ciencias, éste sería el de "cultura"; sin embargo, aclara que este término no significa que haya sido entendido de la misma manera por todos los/las antropólogos / as, sino que ha sido el concepto central y definitorio de la antropología ante las otras ciencias sociales. Añade que uno de sus intereses ha sido esclarecer en qué medida ciertas características y conductas humanas son aprendidas mediante la cultura o están inscritas genéticamente en la naturaleza humana. Dicha interrogante ha llevado a un debate sobre qué es lo determinante en el comportamiento humano, si los aspectos biológicos o los socioculturales, y en especial ha cobrado fuerza respecto a las diferencias entre hombres y mujeres. En los últimos años, las conclusiones han girado alrededor del tema de que las diferencias de género constituyen algunas de las diferencias significativas entre los sexos.

En suma, al hablar de género se centra la atención en aquellas diferencias que han sido atribuidas tradicionalmente a la naturaleza biológica de hombres y mujeres, el lugar de su posición en la estructura social, y las expectativas sociales sobre las cuales se definen los comportamientos adecuados para cada sexo. La llamada perspectiva de género trae al debate cuestionamientos sobre el poder y la igualdad, y se rechaza la idea de que hombres y mujeres son fundamentalmente diferentes en temperamento, habilidades e intereses. ${ }^{5,7,8}$ En otras palabras, se trata de aquella perspectiva donde el género se articula con las modalidades en que una determinada sociedad visualiza la feminidad y la masculinidad, y al utilizar este concepto en lugar de sexo se busca subrayar que lo femenino y masculino no son categorías bipolares ni fijas, pues son construcciones socioculturales. Así pues, el género alude a los mecanismos a través de los cuales las diferencias biológicas se transforman en desigualdades sociales. ${ }^{2}$

Si se parte del concepto de que la salud de la mujer es una síntesis de la biología propia de su sexo y del lugar que la sociedad le asigna en un determinado contexto histórico cultural, se entenderá que ambos factores se integran en el concepto de género, que no sólo se refiere al sexo biológico, sino también a la amplia gama de conductas, expectativas y roles atribuidos a hombres y mujeres por las estructuras sociales. ${ }^{9}$ Los condicionantes biológicos corresponden a la anatomía y a la fisiología 
de la mujer, mientras que los condicionantes sociales y culturales se relacionan con las oportunidades de la mujer de acceder a la educación, la alimentación y los servicios de salud. Los comportamientos y modos de vida de cada uno de los sexos relacionados con la salud, también son elementos centrales de la teoría de género. ${ }^{10}$

En el campo de la salud, Cardaci ${ }^{3}$ señala que, al incorporar el género en sus análisis, diversas investigaciones contribuyen a poner de manifiesto la forma en que esta categoría está presente con relación a los riesgos y protecciones que se toman frente a diversas enfermedades, a la percepción de los síntomas, a la organización y desarrollo de la atención a la salud, a las prioridades para otorgamientos de apoyos financieros, a las preguntas que se hacen los/las investigadores/as y los / las clínicos/as, al diagnóstico de un padecimiento, así como a las decisiones respecto a su tratamiento. En 1993 Gómez ${ }^{11}$ planteaba que algunas diferencias de morbimortalidad entre niños y niñas eran señales de alarma respecto a la posible existencia de un importante problema social: la discriminación en contra de las niñas. No obstante, Cardaci ${ }^{12}$ destaca que la debilidad principal de varias explicaciones reside en el aislamiento de la categoría de género respecto del resto de los factores socioeconómicos, culturales y biológicos que entran en juego en el proceso salud-enfermedad-muerte. Esta autora resalta la necesidad de evitar reduccionismos y de considerar al género como una determinante que no opera aislada de todo un conjunto de variables, sino que es una preocupación presente en diversos estudios publicados por académicos/as de diversos países. En el tema de la salud, Cardaci resume el enfoque de género como una de las vías a través de las cuales es posible incorporar a la discusión nuevos temas y problemas, así como enriquecer aquellas explicaciones sobre la morbimortalidad que no se sustentan exclusivamente en el nivel biológico, sino que otorgan un papel central a los factores socioculturales y psicológicos.

Ahora bien, las relaciones entre los géneros conforman una de las dimensiones sociales más importantes en relación con la salud y nutrición de la mujer. La desigualdad entre hombres y mujeres se expresa en diferencias en el acceso a recursos, los grados de autonomía personal derivados del papel que desempeñan en la familia y en la sociedad, la valoración y retribución social del trabajo e, incluso, la capacidad de decidir, planear y disfrutar la propia vida.,3,8-10,12 En este sentido, las contradicciones en las relaciones entre los géneros, no son producto de diferencias biológicas propias de cada sexo, sino de construcciones sociales y simbolizaciones culturales de los sistemas de género/sexo que las sociedades elaboran a partir de las diferencias anatomo- fisiológicas.

Antes de entrar al siguiente apartado, cabe subrayar que el uso adecuado del enfoque de género como herramienta analítica, lejos de "cortar" la realidad, enriquece los marcos teóricos explicativos del proceso salud-enfermedad-atención al develar algunas situaciones empíricas entre hombres y mujeres como son: a) necesidades especiales de atención, aun en ciertos casos de condiciones que afectan a ambos sexos; $b$ ) riesgos específicos ligados a actividades o tareas definidas como masculinas o femeninas; c) percepciones de enfermedad; d) conductas de búsqueda de atención; e) grado de acceso y de control ejercido por las personas sobre los recursos básicos para la protección de la salud, en los planos tanto intrafamiliar como público; y, f) prioridades en la distribución de recursos públicos destinados a la provisión de medios y cuidados para la salud, y a la investigación sobre los problemas de salud que afectan de manera diferente o exclusiva a uno de los sexos. ${ }^{11}$

\section{Las mujeres y los estudios de alimentación y nutrición: una mirada hacia atrás}

Al hacer un recuento sobre la incorporación de la teoría y perspectiva de género en los estudios de alimentación y nutrición en México, se observa que ésta es escasa: por un lado se encuentran sólo unos pocos datos desagregados por sexo, lo que no permite extraer conclusiones definitivas, y por el otro, se aprecia una reiterada confusión entre lo que es género y lo que es sexo. No obstante, en los últimos años se ha venido destacando con creciente interés la necesidad de realizar análisis que tomen en cuenta la problemática de los géneros. A continuación se revisarán algunos trabajos.

La preocupación por estudiar la situación nutricia de los países en América Latina está presente desde hace varias décadas, sobre todo en México donde, a partir de 1957 y de una manera sistematizada, el Instituto Nacional de Nutrición, ahora llamado Instituto Nacional de Ciencias Médicas y Nutrición Salvador Zubirán, ha realizado una serie de encuestas en los planos local y nacional. Por otra parte, el Hospital Infantil de México, a través de su Centro Rural en el estado de Morelos, también fue precursor de la investigación epidemiológica de la nutrición.

Durante los primeros años, las encuestas apuntaron a conocer la magnitud de la situación nutricia infantil y preescolar del país y a determinar las principales zonas geográficas con mayores problemas. Fue así como se identificó al sureste de la República mexicana y algunas 
regiones del centro, como las áreas más afectadas por la desnutrición. Las prácticas alimentarias estudiadas en las primeras encuestas de nutrición se reducían a investigar la duración de la lactancia materna, la edad de la ablactación, los alimentos más utilizados en este periodo y la edad del destete. Los instrumentos de recolección y las técnicas utilizadas en estas encuestas, desde finales de los años cincuenta hasta principios de los setenta, fueron las de "pesas y medidas" de siete y tres días y las cualicuantitativas de registro de consumo de alimentos en las últimas 24 horas en los ámbitos familiar y preescolar, aunadas al estudio de algunos signos clínicos en la población menor de cinco años. Para 1974, se habían realizado más de 35 encuestas en la República mexicana. ${ }^{13,14}$ Desde el punto de vista metodológico, la información derivada de estos estudios tenía la limitante de que se desconocía, con excepción de la alimentación del/de la niño/a entre 1 y 5 años, el consumo real de cada uno de los miembros de la familia; lo que se sabía era únicamente la ingesta promedio per cápita diaria. Asimismo, los aspectos clínicos, bioquímicos y antropométricos de la población infantil también se estudiaban, aunque rara vez se analizaban según el sexo.

Entre 1974 y 1996 se realizaron varias encuestas nacionales de alimentación en comunidades rurales ${ }^{15-17}$ y aunque en la de 1996 sí se investigó el consumo de alimentos de las mujeres, el tipo de análisis efectuado no difirió en absoluto al de las encuestas anteriores, ya que sólo se limitó a describir las cantidades de los alimentos y de los nutrimentos consumidos, así como los porcentajes de adecuación con respecto a sus recomendaciones. La Encuesta Urbana de Alimentación de $1995,{ }^{18}$ que recabó información tanto de hombres como de mujeres, mostró que el sexo masculino, entre los 18 y los 39 años y de 50 a 69 años, se encontraba ligeramente en mejor situación nutricia que el femenino.

Por su parte, la Secretaría de Salud, junto con el Instituto Nacional de Salud Pública han llevado a cabo varias encuestas nacionales de nutrición. ${ }^{19-21}$ La encuesta de 1999 ofrece datos desagregados sobre el estado de nutrición de los preescolares con base en la estatura para la edad, como indicador de desnutrición crónica, además de que permite evaluar discriminación en términos de alimentación. Esta última información, al ser interpretada a través de una visión de género, permite concluir que, de acuerdo con el indicador talla y peso para la edad, "los niños y niñas en México se encuentran en condiciones semejantes de nutrición y no existen evidencias para pensar en una discriminación en contra del género femenino" ${ }^{9,20}$ Resalta la presencia de sobrepeso y obesidad entre el grupo femenino debido a patrones de alimentación inadecuados, aunados a hábitos como el tabaco y la vida sedentaria de las mujeres mexicanas, que propician la aparición de enfermedades crónicas.

En cuanto a la información derivada de la ENSANUT $2006,{ }^{21}$ la relacionada con la población preescolar hasta el momento no se encuentra desagregada por sexo, a diferencia de los datos de la población escolar, adolescente y adulta. La prevalencia de baja talla en niños de 5 a 11 años de edad del sexo masculino fue de $10.4 \%$ y del femenino de $9.5 \%$ y la prevalencia de sobrepeso y obesidad comparada con los datos de 1999, aumentó en ambos sexos de manera similar. Por lo que respecta a los datos de sobrepeso y obesidad de las y los adolescentes, no presentaron grandes diferencias, ya que la población femenina registró 23.3 y $9.2 \%$, respectivamente, y la masculina, 21.2 y $10.0 \%$. La prevalencia de sobrepeso fue más alta en hombres que en mujeres mayores de 20 años; en cambio la de obesidad fue mayor en mujeres. Al sumar las dos prevalencias, se observa que las mujeres (71.9\%) padecen más estas enfermedades que los hombres $(66.7 \%)$. Aun cuando no se haya realizado todavía un análisis con perspectiva de género, se considera como un gran avance la desagregación de los datos según sexo de las diversas encuestas nacionales de nutrición y de alimentación. El adentrarse en lo que hay más allá de los datos es una tarea pendiente para entender cuáles son los determinantes de las altas prevalencias de sobrepeso y obesidad entre las mujeres.

De una manera paralela a la realización de estas encuestas, se llevaron a cabo otras investigaciones en México que, a diferencia de las nacionales, se abocaron a estudiar el estado nutricio de la población infantil, el valor nutritivo de su ingesta habitual, la correlación del estado de nutrición con el desarrollo psicomotor, los factores del medio ambiente asociados con la desnutrición, y algunos conceptos existentes en la población materna acerca de la causalidad de algunas enfermedades, entre otros, en algunas comunidades rurales del país. Destacan los estudios longitudinales de crecimiento y desarrollo de Chávez ${ }^{22}$ y Cravioto, ${ }^{23}$ pioneros de estas investigaciones en México y que a fines de los años sesenta echaron mano de dos estrategias principales: los estudios multifactoriales y los de observación clínica y experimental, con énfasis en factores individuales específicos. En esas investigaciones algunos datos fueron desagregados por sexo.

A fines de los años sesenta y hasta mediados de la década de los ochenta, también se dio el auge de los estudios sobre lactancia materna. Los abordajes teóricos de las investigaciones fueron variados, pues además de continuar con la visión biologicista de la lactancia materna centrada en el/la recién nacido/a, esto es, su crecimiento y desarrollo, se realizaron estudios y 
escribieron ensayos, cuyo marco teórico se derivó de las ciencias sociales, sobre todo de la sociología y la antropología. En estos estudios el sujeto de análisis continuó siendo el hijo o la hija; sin embargo, en la búsqueda de explicaciones que permitieran dar respuesta al por qué del abandono progresivo del amamantamiento en la década de los años setenta, las teorías de la multicausalidad basadas en los "círculos viciosos de la desnutrición", cobraron importancia.

Es en ese período cuando nuevos modelos teóricos -por ejemplo, el Modelo Médico Hegemónico-, dieron otro giro a las investigaciones sobre lactancia, desde la formulación de las interrogantes, de los problemas, y la forma de abordarlos, hasta en el análisis de los datos. ${ }^{24,25}$ La mujer continuó siendo el foco de atención en este tipo de estudios en tanto madre -vehículo o instrumento- del recién nacido, pero a diferencia de las teorías de la multicausalidad basadas en los círculos viciosos, se estudiaron y analizaron otros elementos como el económico-político, el institucional, y la práctica y el saber médico para la explicación de las prácticas y representaciones maternas. Lo anterior permitió una deconstrucción de la lactancia como un espacio predominantemente biológico y psicológico y, a la vez, una reconstrucción de esta temática como un objeto de estudio más globalizador e integrador. Finalmente, desde mediados de los años ochenta la perspectiva de género se incorporó como elemento explicativo de la práctica de lactancia materna y de su abandono. ${ }^{26-28}$

Aunado a lo anterior, en los últimos años también se han llevado a cabo investigaciones relacionadas con el consumo de alimentos y de nutrimentos en las diferentes etapas del ciclo vital, en las que se incorpora ya no sólo la explicación biológica del fenómeno, sino, además, una interpretación social en donde el género se aborda como una determinante que se relaciona con otras variables e indicadores. Los hallazgos de estos estudios presentan cierta similitud: por ejemplo, en las comunidades estudiadas no se han encontrado diferencias en la distribución y consumo de alimentos entre mujeres y hombres adultos y preescolares, ${ }^{29-32}$ y las conclusiones giran en torno a que los bajos consumos de alimentos, energía y nutrimentos son más atribuibles a las condiciones de pobreza en las comunidades que a una distribución inequitativa de los alimentos al interior de la familia. También existen otras investigaciones que, al pretender identificar la magnitud del daño de la población vulnerable -ninos/as y mujeres-, y relacionarla con algunos factores socioculturales condicionantes, entre ellos la diferencia y desigualdad genérica, se limitan a clasificar el estado nutricio de las mujeres y hacen a un lado el análisis de género. ${ }^{33}$

\section{¿Qué falta por hacer?}

Ya se habló brevemente sobre el recorrido de algunos estudios alimentarios y nutricionales en México y es innegable la relevancia de los diversos acercamientos teóricos y la importancia que han tenido en el conocimiento de la nutrición; empero, cabe insistir en la perspectiva de género como otro elemento central en el análisis de los datos sobre alimentación, no sólo de la población femenina, sino también de la masculina. Se parte del hecho de que los temas a estudiar son prácticamente los mismos; sin embargo, los supuestos teóricos, las preguntas de investigación, la forma de abordarlas y de analizar la información, son diferentes.

Es un hecho que, desde el punto de vista biológico, existen diferencias en los requerimientos de energía y de nutrimentos entre hombres y mujeres; está claro que, en general y haciendo a un lado aquellos casos de mujeres que tienen una gran actividad física o que se encuentran en los periodos de embarazo y de lactancia, durante los cuales se requiere aumentar el consumo de energía y de algunos nutrimentos, los hombres necesitan comer más. Sin embargo, adentrarse en las prácticas, hábitos, costumbres alimentarias y significados de los alimentos y del acto de comer de las familias, implica un abordaje teórico-metodológico diferente al que se lleva a cabo en el campo de la nutrición. Los seres humanos comen alimentos y, como afirma De Garine, ${ }^{34}$ el terreno de la alimentación es un campo de elección en el que se revela la estructura profunda de la sociedad. La comida y sus significados son un buen ejemplo de las diferencias (igualdades e inequidades) entre ambos sexos, donde los temas de autoridad y poder son cruciales.

En este sentido la alimentación constituye uno de los pocos terrenos en que un fenómeno es relevante a la vez tanto para las ciencias biológicas como para la sociales y la discusión teórica y las evidencias empíricas sobre el estado nutricio de las mujeres y de los hombres son un marco propicio para la incorporación de nuevos planteamientos conceptuales desarrollados por las ciencias sociales. Tal es el caso de la perspectiva de género que ofrece alternativas para contribuir a la explicación de los problemas de nutrición de las mujeres y de los hombres. La incorporación de la categoría de género en las investigaciones ha permitido abordar, mas no resolver, los niveles de complejidad que se conforman entre lo masculino y lo femenino. No obstante, se considera como un gran reto incluir esta perspectiva que ofrece otro tipo de conocimientos en la temática de la alimentación y nutrición individual y familiar.

Problematizar las condiciones de nutrición de las mujeres y de los hombres y explorar con más detalle los diversos factores que intervienen, mantienen o modi- 
fican su salud, particularmente su estado nutricio ,son algunas líneas de investigación y de acción que pueden seguir las y los interesados en el tema de la nutrición con una perspectiva de género. Sin embargo, desde el punto de vista antropológico el uso de esta categoría implica, entre otras cosas, introducirse al conocimiento sobre cómo el acto de comer dentro de las familias, y el proceso alimentario -decisión, provisión o adquisición, preparación y distribución de alimentos-adquieren relevancia desde un punto de vista social y si éstos, además de marcar diferencias sexuales, tiene una significación genérica. Cabe recapacitar en este punto.

Si se parte de lo anterior, re-pensar en las mujeres y en los hombres durante los hechos relacionados con la alimentación sería, entre otros aspectos, hacerlas / os visibles en situaciones histórico concretas, principalmente como mujeres y hombres y no únicamente en su papel de madres-reproductoras y de padres-proveedores. No se discute el hecho de que, tradicionalmente, las mujeres han sido y continúan siendo las responsables de la salud familiar y las "beneficiarias" o "depositarias" de algunas de las principales acciones del sector salud, además de que, por encontrarse en el ámbito doméstico, también sean ellas quienes proporcionen información sobre lo que ocurre dentro de sus familias; no obstante, reconstruirlas como mujeres es algo más que eso.

Esta imposibilidad de dar visibilidad a las mujeres, y también a los hombres, en la mayoría de los estudios alimentarios y nutricionales, ha motivado a algunas/os investigadoras/es interesadas/os en el tema de la salud de la mujer, a reflexionar acerca de cuál sería el camino más viable para hacer visibles a las mujeres en este campo e incursionar en investigaciones sobre la salud y nutrición femenina bajo una perspectiva de género. Así entonces, algunas de las interrogantes que surgen son las siguientes: ¿mientras que en el campo de la nutrición se elabora una teoría o conjunto de teorías sobre la nutrición y su relación con el género, por qué razón no se retoma parte de la experiencia que han sistematizado las y los especialistas en el campo de la salud reproductiva y mental sobre el cuerpo vivido y la experiencia vivida? Si los procesos individuales biológicos de la nutrición atraviesan el propio cuerpo y las prácticas y representaciones sobre la alimentación se conforman dentro de un marco socioeconómico y cultural y, sobre todo, por la experiencia vivida de las mujeres, ¿por qué no comenzar a investigar, reflexionar, buscar explicaciones, orientar, capacitar y educar, bajo la premisa de que la experiencia de las mujeres es fundamental para comprender qué sucede con ellas, con sus prácticas alimentarias, con las de sus parejas y sus familias y, por consiguiente, tener elementos para cambiar su situación si es que debe cambiarse? En lo que respecta a la población masculina, ¿por qué no adentrarnos en la alimentación y el estado nutricio de los hombres que viven en las comunidades rurales y su relación con ciertas prácticas "problemáticas", por ejemplo, el excesivo consumo de alcohol?

Antes de finalizar r este trabajo cabe compartir algunas reflexiones, sobre el tema de la lactancia materna. La investigación se llevó a cabo con mujeres indígenas de la Sierra Juárez en el estado de Oaxaca; ${ }^{26}$ el eje rector fue el género, y el abordaje las técnicas cualitativas. Los objetivos e interrogantes fueron distintos a los planteados en los años sesenta y setenta, ya que a diferencia de aquellos estudios donde la población menor de un año era el centro de atención en cuanto a su estado nutricio, crecimiento y desarrollo, el estudio aludido partió de categorías y variables socioculturales tales como saber popular y saber científico acerca de la alimentación de la población infantil; tradiciones y consejos familiares; atención del parto; autoatención; "rol" de las parteras $\mathrm{y}$ de las mujeres y hombres de la familia en el proceso alimentario; experiencia vivida de las mujeres-madres; prácticas y representaciones sobre el amamantamiento; preferencias y restricciones alimentarias durante la lactancia; apoyos y redes familiares y presencia paterna, lo que dio lugar a otro tipo de conocimientos y, por lo tanto, de explicaciones e interpretaciones.

Con base en lo anterior, cuando se realizaron las entrevistas relacionadas con la práctica de lactancia en un grupo de mujeres indígenas, lo primero que se observó fue que los infantes varones recibían por más tiempo la leche de las madres. Uno de los aspectos que llamaron la atención fue el significado de fuerza de la leche materna: "los niños necesitan tomar más tiempo leche de sus madres para tener más fuerza y así estar más sanos para trabajar en el campo y mantener a su familia", a diferencia de las niñas que permanecen en la casa ayudando a sus madres en las labores de la casa. En otras palabras, los sucedáneos de leche materna y el trabajo doméstico tienen menor valor en comparación con el trabajo masculino. Lo anterior, entre otros muchos factores, muestra situaciones y prácticas alimentarias diferenciales según género en la población materna entrevistada en Oaxaca, que no habrían salido a relucir dei no adentrarse las investigadoras en las diferencias sexuales y la perspectiva de género. Sin embargo, cabe destacar que no se encontraron prácticas desiguales en el cuidado de las niñas y los niños, pues ambos sexos reciben la misma atención cuando se enferman. Salas ${ }^{28}$ sintetiza la importancia de conceptualizar la lactancia de una manera distinta, señalando que, "caminar por la acera de enfrente consiste en visualizar que la lactancia materna no es un asunto del sexo femenino, es un asunto 
de género, es decir, corresponde a uno de los atributos asignados socialmente a partir de una diferenciación biológica inicial".

Por último, asumir la perspectiva de género en las acciones de investigación alimentaria y nutricia, implica pensar tanto en las mujeres como en los hombres, así como en las formas de relación entre ellos. La ubicación dentro de esta perspectiva permite conocer cuáles son los recursos y la capacidad de acción de cada sexo para encarar las dificultades de la vida y realizar sus propósitos. Partir de la identificación de los problemas y necesidades de los hombres y de las mujeres, entre los que deben considerarse los relacionados con la desigualdad entre los géneros, para incorporarlos en el conjunto de aspectos que se van a cubrir, es otro de los objetivos de los proyectos. Ciertamente el reto es difícil, y un primer paso consiste en incorporar la perspectiva de género como parte de los currículos académicos de las diferentes escuelas y facultades de salud, como las de medicina, enfermería y nutrición en el país y, en las instituciones dedicadas a la investigación, promover la aplicación de la perspectiva de género y las técnicas cualitativas, en combinación con las cuantitativas, para profundizar en las diferencias y desigualdades según sexo/género en el ámbito de la alimentación y nutrición.

\section{Referencias}

I. Cerón-Mireles P, Sánchez-Carrillo C, Robledo Vera C, del Río Zolezzi A, Pedroza-lslas L, Reyes -Zapata H, et al. Aplicación de la perspectiva de género en artículos publicados en cuatro revistas nacionales de salud, 2000-2003. Salud Publica Mex 2006;48(4):332-340

2. Ravelo P, Ramírez JC. Prólogo. En: Pérez Gil SE, Ramírez JC, Ravelo P, coords. Género y salud femenina. Experiencias de investigación en México. México: CIESAS-U de G-INNSZ, 1995: I I-19.

3. Cardaci D. El enfoque de la salud desde el género. Rev Mujer Salud. RSMLAC. 1999;75:3-4

4. Basaglia F. Mujer, locura y sociedad. México: Universidad de Guadalajara, 1986.

5. Lamas M. Usos, dificultades y posibilidades de la categoría género. En: Lamas M, comp. El género. La construcción cultural de la diferencia sexual. México: PUEG-UNAM-Porrúa, 1996:327-366.

6. Scott J. El género, una categoría útil para el análisis histórico. En: Lamas $M$, comp. El género: la construcción cultural de la diferencia sexual. México: PUEG-UNAM-Porrúa, 1996:265-302.

7. Goldsmith M. Debates antropológicos en torno a los estudios sobre la mujer. Nueva Antropología. 1986;VIII(30):I47-I7I.

8. Sigel R.Ambition and accomodation. How women view gender relations. Chicago:The Univ. of Chicago Press. Citado en: D. Cardaci. El enfoque de la salud desde el género. Rev. Mujer Salud. RSMLAC. 1999; 3-4: 72
9. Langer A, Lozano R. I. Condición de la mujer y la salud. En: Figueroa JG, comp. La condición de la mujer en el espacio de la salud. México: Colmex, 1998:33-82

10. Rathgeber E,Vlassof C. Gender and tropical diseases: a new research focus. Ginebra: OMS, 1992. Citado en:A. Langer y R Lozano R. I.

Condición de la mujer y la salud. En: Figueroa JG, comp. La condición de la mujer en el espacio de la salud. México: Colmex, 1998:33-82.

II. Gómez E. Discriminación por sexo y sobremortalidad femenina en la niñez. En: Gómez E, ed. Género, mujer y salud.Washington: OPS. Publ. Núm. 54I, 1993.

12. Cardaci D. Salud, género y programas de estudios de la mujer en México. México: PUEG-UNAM-UAM-OMS, 2004.

13. División de Nutrición. Encuestas nutricionales en México (Encuestas familiares). Volumen I. Estudios de 1958 a 1962. México: Edición L - I. División de Nutrición, 1972.

14. División de Nutrición. Encuestas nutricionales en México (Encuestas familiares). Volumen II. Estudios de 1963 a 1974. México: Edición L - 2 I. CONACYT-PRONAL-División de Nutrición, 1976.

15. Madrigal H, Moreno-Terrazas 0, Chávez A. Encuesta Nacional de Alimentación 1979. México. Publ. División de Nutrición. L - 46, 1982. 16. Madrigal H (1989) ed. Encuesta Nacional de Alimentación en el Medio Rural por Regiones Nutricionales 1989. México. Publ. Div. de Nutr. L - 90 17. Avila A, Shamah T, Chávez A. Encuesta Nacional de Alimentación y Nutrición en el Medio Rural 1996. Resultados por entidad.Volumen I. México: INNSZ-SEDESOL-DIF-SSA-IMSS-INI-UNICEF, 1997.

18. Instituto Nacional de Nutrición Salvador Zubirán. Encuesta Urbana de Alimentación en la Zona Metropolitana de la Ciudad de México. México: INNSZ-Fidelist-DIF-IPC-Foro de Apoyo Mutuo, 1995.

19. Sepúlveda J, Lezana MA, Tapia-Conyer R, Valdespino JL, Madrigal $\mathrm{H}$, Kumate J. Estado nutiicional de preescolares y mujeres en México: Resultados de una encuesta probabilística provisional. Gac Med Mex 1990;126(3):207-225.

20. SSA-INSP-INEGI. Encuesta Nacional de Nutrición. 1999. Estado nutricio de niños y mujeres en México. México: SSA-INSP-INEGI, 2000. 21. Olaiz-Fernández G, Rivera-Dommarco J, Shamah-Levy T, Rojas R, Villalpando-Hernández S, Hernández-Avila M et al. Encuesta Nacional de Salud y Nutrición, 2006. Cuernavaca, México: Instituto Nacional de Salud Pública, 2006.

22. Chávez A, Martínez C. Nutrición y desarrollo infantil. México: Editorial Interamericana, 1979.

23. Cravioto J , Licardie E . Ecology of malnutrition. Pediatr 1979; I4:I57.

24. Arana M. Las fórmulas lácteas para la alimentación infantil: complementariedad entre las compañías monopólicas y las instituciones estatales. Rev Salud Latinoamerican 1982;2: I-6.

25. Ysunza A. El abandono de la lactancia materna en México. II. Su causalidad. Rev Invest Clin 1986;(supl 38):97-102.

26. Pérez-Gil SE, Rueda F, Díez-Urdanivia S. Lactancia y cuidado de los hijos: un estudio comparativo en dos zonas rurales del país. Salud Publica Mex 1993;35(6):692-699

27. Pérez-Gil SE, Díez-Urdanivia S, Pérez L,Valdés S, Gutiérrez G. Embarazo y lactancia: experiencia de un grupo de madres de dos zonas rurales. Rev. Nutrición Clínica 1999;2(3): I I6-126.

28. Salas M. Lactancia materna: las mujeres como protagonistas. En: SE Pérez Gil, JC Ramírez, Ravelo P. Género y salud femenina. Experiencias de investigación en México. México: CIESAS-U de G-INNSZ, 1995:I27-136. 29. Pérez-Gil SE, Díez-Urdanivia S, Pérez L, Gutiérrez G y Valdés $S$. Consumo de energía y de proteínas en mujeres de zonas rurales de México: una aproximación cualitativa y de género. Rev Nutrición Clínica 200I;4(I):4-12.

30. Pérez-Gil SE, Caire P. Diferencias genéricas en la alimentación y nutrición de preescolares en una comunidad rural: una aproximación 
cualitativa. En:A Romero, P Torre,V M Muñoz, D Espinola, coords. Quehacer científico. Un panorama actual en la UAM-Xochimilco.Tomo II. México: UAM-X, 2004:183-204.

31. Gurri F, Farías P. Cambios en la adaptabilidad de niños y niñas durante los primeros diez años de vida en los altos de Chiapas. Rev Salud Problema, 1998;5:4I-50.

32. Cedillo Nakay R, Trujillo-Hernandez B, Huerta M,Trujillo X,Vazquez

$C$. Prevalencia de desnutrición por sexo y por grupos de edades en preescolares de familias con bajos ingresos del estado de Colima. Bol Med Hosp Infant Mex 2002;59:615-625.

33. Backstrand J,Allen L, Pelto G, Chávez A . Examining the gender gap in nutrition: an example from rural Mexico. Soc Sci Med 1997;44:175I-1759.

34. De Garine I.Antropología de la alimentación y pluridisciplinariedad.

Am Indig 1988;XLVIII(3):635-650. 